\title{
CRISPR Cas9 in Pancreatic Cancer Research
}

\author{
Hai Yang, Peter Bailey and Christian Pilarsky* \\ Department for Surgical Research, Universitätsklinikum Erlangen, Erlangen, Germany
}

Pancreatic cancer is now becoming a common cause of cancer death with no significant change in patient survival over the last 10 years. The main treatment options for pancreatic cancer patients are surgery, radiation therapy and chemotherapy, but there is now considerable effort to develop new and effective treatments. In recent years, CRISPR/Cas9 technology has emerged as a powerful gene editing tool with promise, not only as an important research methodology, but also as a new and effective method for targeted therapy. In this review, we summarize current advances in CRISPR/Cas9 technology and its application to pancreatic cancer research, and importantly as a means of selectively targeting key drivers of pancreatic cancer.

Keywords: pancreatic cancer, CRISPR/Cas9, gene editing, knock out and knock in, library screening

\section{OPEN ACCESS}

Edited by:

Vincenzo Corbo,

University of Verona, Italy

Reviewed by:

Michael E. Feigin,

Watson School of Biological

Sciences, United States

Serif Senturk,

Dokuz Eylül University, Turkey

*Correspondence:

Christian Pilarsky

christian.pilarsky@uk-erlangen.de

Specialty section:

This article was submitted to

Stem Cell Research,

a section of the journal

Frontiers in Cell and Developmental

Biology

Received: 12 July 2019

Accepted: 01 October 2019

Published: 18 October 2019

Citation:

Yang $H$, Bailey $P$ and Pilarsky $C$ (2019) CRISPR Cas9 in Pancreatic

Cancer Research.

Front. Cell Dev. Biol. 7:239.

doi: 10.3389/fcell.2019.00239

\section{INTRODUCTION}

According to the data provided by the North American Association of Central Cancer Registries (NAACCR), pancreatic cancer is the 12 th most common cancer with the 5th worst prognosis in the United States. It is estimated that there will be 56,770 new cases of pancreatic cancer and 45,750 pancreatic cancer-related deaths in the United States in 2019. Compared to other common cancers, pancreatic cancer has not seen a significant improvement in patient outcomes, with a $9 \%$-year relative survival (Siegel et al., 2019). Surgery, radiation therapy and chemotherapy are still the main treatment options for pancreatic cancer, but there is now considerable effort in identifying better treatment strategies for pancreatic cancer, such as targeted therapy, immune therapy and potentially CRISPR/Cas9 directed gene therapy.

Currently CRISPR/Cas9 (clustered regularly interspaced short palindromic repeats and CRISPR-associated protein 9) is emerging as a powerful gene-editing tool with potential in precision medicine. CRISPR repetitive sequences were first observed by Ishino et al. (1987), with subsequent work performed by Jinek et al. (2012) proving that an endonuclease can be directed to cleave target DNA by a two-RNA structure. Since then, CRISPR/Cas9 technology has rapidly evolved, driving incredible progress in research and clinical applications. Compared to the other gene-editing technologies, such as meganucleases (MNs), zinc finger nucleases (ZFNs) and transcription activator-like effector nucleases (TALENs), CRISPR/Cas9 technology has lower cost, higher efficiency and is less complex in its application (Osborn et al., 2016).

\section{BACKGROUND OF CRISPR/CAS9}

CRISPR (clustered regularly interspaced short palindromic repeats) were first described in 1987 by Ishino et al. (1987) who identified highly conserved nucleotide sequences exhibiting a 14 bp dyad symmetry at the $3^{\prime}$-end flanking region of the isozyme-converting alkaline phosphatase (iap) gene 
in Escherichia coli. However, the function and biological importance of CRISPR sequences were not fully understood until 2007, when Barrangou et al. (2007) exposed Streptococcus thermophilus to phage and sequenced the resultant phageresistant variants. Analysis of the variant DNA revealed that the bacteria had gained new CRISPR spacers that were derived from the phage genome. The identification of CRISPR sequences in bacterial genomes subsequently led to the identification of a set of homologous genes referred to as CRISPR and associated (cas) genes that together comprise the CRISPR locus. The insertion of CRISPR-Cas in the genomes of bacteria infected with virus suggested that CRISPR-Cas may provide resistance against phages and that resistance to infection might be enhanced or decreased by inserting or deleting spacer-phage sequence.

Jinek et al. (2012) in 2012 were the first to demonstrate that the CRISPR-Cas system could produce a mature CRISPR RNA (crRNA) and base-paired trans-activating crRNA (tracrRNA) that together form a two-RNA hybrid structure. This two-RNA structure could guide CRISPR-associated protein Cas9 to specific DNA sequences and generate double-stranded (ds) breaks in target DNA. In 2013, Cong et al. (2013) and Mali et al. (2013) successfully edited the DNA sequences of the EMX1, PVALB, PPP1R12C genes in human and the Th gene in mouse using the CRISPR/Cas9 system. This was the first demonstration of the power of the CRISPR-Cas9 system to edit the genomes of eukaryotic cells. Since then, a wealth of research has improved the CRISPR/Cas9 gene editing tool's specificity, orthogonality and multiplexibility in various species, and has led to the development of new omics applications.

CRISPR/Cas9 technology has been rapidly promoted and applied in the: generation of animal models; gene function research; multiplexed mutations; and chromosome rearrangements. Compared to traditional gene editing tools such as MNs, ZFNs, TALENs and so on, CRISPR/Cas9 technology provides the advantage of lower cost, higher efficient and greater simplicity (Osborn et al., 2016).

\section{APPLICATION OF CRISPR/CAS9 IN PANCREATIC CANCER}

\section{CRISPR/Cas9 Gene Editing in Pancreatic Cancer}

An homologous guide RNA can guide the cas9 nuclease to target a specific site in the genome. When cas9 is guided to the target DNA sequence and there is a Protospacer Adjacent Motif (PAM) sequence downstream, it will cut both strands of the genome at that location. The cells then repair the break using two distinct mechanisms: (1) imprecisely by using the non-homologous end joining (NHEJ) repair pathway, which causes the insertion or deletion of bases; or (2) accurately by the homology-directed repair (HDR) pathway. Based on existing homologous DNA sequences, the HDR pathway can repair DNA damage accurately by mediating a strand-exchange process involving the provided DNA sequence as a repair template to insert a matching DNA sequence into the break.
By inducing NHEJ or HDR, CRISPR/Cas9 knockouts or knockins can be achieved by deleting, replacing or adding genetic sequences.

CRISPR/Cas9 system is widely used in pancreatic cancer research to knock-out genes implicated in disease progression. Watanabe et al. (2018) used CRISPR/Cas9 to knock out KDM6A in human Pancreatic ductal adenocarcinoma (PDAC) cell lines to demonstrate that KDM6A-deficient cells exhibit an aggressive phenotype. Pessolano et al. (2018) and Belvedere et al. (2016) showed that, CRISPR/Cas9-directed knock-out of ANXA1 in Mia $\mathrm{PaCa} 2$ cells, resulted in the secretion of fewer extracellular vesicles and a weak motile phenotype. After knock out of GALNT3 in Capan 1 cells, Barkeer et al. (2018) found that cells formed fewer tumorspheres, lost their ability to self-renewal, and migrate. Yuza et al. (2018) demonstrated that knock out of SphK1in PAN02 cells resulted in increased proliferation and migration. C1GALT1 was knocked out of PDAC cells by Chugh et al. (2018) with CRISPR/Cas9 technology, and the cells showed increased growth, migration, tumorigenicity, metastasis and expression of Tn and sTn. There are also some other researches in pancreatic cancer based on the same strategy by knocking out specific gene of pancreatic cancer cells, then observe the changes in different phenotypes (Table 1; Belvedere et al., 2016; Hesler et al., 2016; Muniyan et al., 2016; Rao et al., 2016; Vorvis et al., 2016; Ayars et al., 2017; Harada et al., 2017; He et al., 2017; Lal et al., 2017; Muzumdar et al., 2017; Yu et al., 2017; Zhang et al., 2017; Barkeer et al., 2018; Chugh et al., 2018; Liu et al., 2018a,b, 2019; Pessolano et al., 2018; Santoro et al., 2018; Wang et al., 2018; Watanabe et al., 2018; Yasunaga et al., 2018; Yuza et al., 2018; Abdalla et al., 2019; Hwang et al., 2019; Li et al., 2019b,c; Stock et al., 2019). Collectively, these studies show that CRISPR/Cas9 is a very powerful gene editing tool for exploring the function of defined genes in cell signaling pathways, proliferation, migration, invasion and potentially chemotherapy resistance of pancreatic cancer. By using CRISPR/Cas9 technology to knock out target genes and characterize phenotypic changes, we can develop a better understanding of biological roles played by target genes, identify potential treatment targets and/or better treatment strategy for pancreatic cancer. But there is still a long way to go before CRISPR/Cas9 technology can be used as an effective treatment strategy in the clinic.

Gene knockins by CRISPR/Cas9 are also quite important for exploring gene functions in tumorigenesis and development and commonly used for building specific gene expression stem cells and animal models in recently years (Yang et al., 2013; Platt et al., 2014; Ma et al., 2017; Li et al., 2019a). But due to the complicated procedure and time consumption, it is still more common to generate gene expressing cells by building gene expression vectors and transducing the vector into cells via retrovirus or lentivirus in pancreatic cancer research (Li et al., 2019c; Sharma et al., 2019; Watanabe et al., 2019).

\section{CRISPR Pooled Library Screening in Pancreatic Cancer}

Using a sgRNA library to perform loss-of-function (LoF) phenotypic screens is a powerful way to identify novel protein 
TABLE 1 | Recent researches of gene function in pancreatic cancer by CRISPR/Cas9 knockouts of pancreatic cancer cells.

\begin{tabular}{|c|c|c|c|}
\hline sgRNA target & Cell lines & Effect & References \\
\hline KDM6A & BXPC3, PANC1 & proliferation $\uparrow$, migration $\uparrow$, invasion $\uparrow$ & Watanabe et al., 2018 \\
\hline ANXA1 & MIA PaCa-2 & migration $\downarrow$, invasion $\downarrow$, extracellular vesicles $\downarrow$ & $\begin{array}{l}\text { Pessolano et al., 2018; } \\
\text { Belvedere et al., } 2016\end{array}$ \\
\hline SphK1 SphK2 & PAN02 & $\begin{array}{l}\text { proliferation } \uparrow \text {, migration } \uparrow \text {, survival of mice } \downarrow \text { proliferation } \downarrow \text {, migration } \downarrow \text {, } \\
\text { survival of mice } \uparrow\end{array}$ & Yuza et al., 2018 \\
\hline GALNT3 & Capan1 & proliferation $\downarrow$, migration $\downarrow$ & Barkeer et al., 2018 \\
\hline C1galt1 & KPC, KPCC & proliferation $\uparrow$, migration $\uparrow$, tumorigenicity $\uparrow$, metastasis $\uparrow$ & Chugh et al., 2018 \\
\hline ARID1A & HPDE & H2K27ac marks $\uparrow$ & Wang et al., 2018 \\
\hline GPRC5a & MIA PaCa-2, TB32047 & proliferation $\downarrow$, migration $\downarrow$, chemotherapy resistance $\downarrow$ & Liu et al., 2018a \\
\hline ECT2 & MIA PaCa-2 & proliferation $\downarrow$, migration $\downarrow$ & Liu et al., 2018b \\
\hline ATG12 & MIA PaCa-2, AR42J & expression of duct cell markers $\uparrow$, expression of acinar cell markers $\downarrow$ & Yasunaga et al., 2018 \\
\hline KRAS & $\begin{array}{l}\text { A13,8988T, PANC1, KP-4, } \\
\text { MM1402, PACO9, PACO19 }\end{array}$ & exhibit PI3K dependence & Muzumdar et al., 2017 \\
\hline \multirow[t]{3}{*}{ HuR } & MIA PaCa-2 & apoptosis $\uparrow$, unable to engraft tumors in vivo & Lal et al., 2017 \\
\hline & Hs $776 \mathrm{~T}$ & unable to engraft tumors in vivo & \\
\hline & HCT116 & proliferation $\downarrow$ & \\
\hline Rab11-FIP4 & PANC-1 & proliferation $\downarrow$, invasion $\downarrow$, metastasis $\downarrow$ & He et al., 2017 \\
\hline IRAK4 & PANC-1, Pa01c & anchorage-independent (Al) growth $\downarrow$ & Zhang et al., 2017 \\
\hline CYR61 & MIA PaCa-2, PANC1 & $\begin{array}{l}\text { hENT1 and hCNT3 expression } \uparrow \text {, cellular uptake of gemcitabine } \uparrow \text {, } \\
\text { gemcitabine-induced apoptosis } \uparrow\end{array}$ & Hesler et al., 2016 \\
\hline MUC16 & Capan1 & $\begin{array}{l}\text { expression of Thomsen-Friedenreich (TF/T) and Thomsen-nouvelle (Tn) } \\
\text { carbohydrate antigens } \downarrow\end{array}$ & Muniyan et al., 2016 \\
\hline FOXA2 & PANC-1 & tumorigenicity $\uparrow$, aggressiveness $\uparrow$ & Vorvis et al., 2016 \\
\hline GCNT3 & MIA PaCa, BxPC-3, PANC-1 & proliferation $\downarrow$, spheroid formation $\downarrow$ & Rao et al., 2016 \\
\hline CTTN & PANC-1, BxPC-3 & migration $\downarrow$, invasion $\downarrow$ & Stock et al., 2019 \\
\hline CRABP-\| & PANC-1 & migration $\downarrow$, invasion $\downarrow$, expression of interleukin 8 (IL-8), MMP-2 and MMP-14 $\downarrow$ & Yu et al., 2017 \\
\hline $\mathrm{HO}-1$ & Capan-1 & Under hypoxia, proliferation $\downarrow$, sensitivity to gemcitabine $\uparrow$ & Abdalla et al., 2019 \\
\hline WNT5B & PANC-1 & migration $\downarrow$, invasion $\downarrow$ & Harada et al., 2017 \\
\hline $\mathrm{CDH} 17$ & Panc02-H7 & Proliferation, colony formation and motility $\downarrow$ & Liu et al., 2019 \\
\hline $\mathrm{El} 24$ & MIA PaCa-2 & Proliferation $\downarrow$ & Hwang et al., 2019 \\
\hline $\mathrm{HO1}$ & $\begin{array}{l}\text { CD18/HPAF, COLO 357, Capan-1, } \\
\text { Mia PaCa-2 }\end{array}$ & under hypoxia, proliferation $\downarrow$, sensitive to gemcitabine $\uparrow$ & Abdalla et al., 2019 \\
\hline IL2RG & TB32043, bkpc58 & Tumor growth in mice $\downarrow$, JAK3 expression in orthotopic tumors $\downarrow$ & Ayars et al., 2017 \\
\hline $\mathrm{HIF}-1 \alpha$ & BxPC-3 & Metastasis $\downarrow$ & Li et al., 2019b \\
\hline MEKK3 & Panc-1, AsPC1 & EMT and cell migration $\downarrow$, tumor growth $\downarrow$, mice overall survival $\uparrow$ & Santoro et al., 2018 \\
\hline ERBB2 & $\begin{array}{l}\text { HPAFII, CAPAN1, Mia PaCa-2 } \\
\text { (all ERBB2-amplified) }\end{array}$ & $\begin{array}{l}\text { ERBB2 signaling, colony formation, anchorage-independent growth and tumor } \\
\text { xenograft formation } \downarrow\end{array}$ & Li et al., 2019c \\
\hline
\end{tabular}

$\uparrow$, up-regulation; and $\downarrow$, down-regulation.

functions in specific growth conditions. The main strategy of CRISPR pooled library screening involves the use of thousands of plasmids in one CRISPR pooled library, with each plasmid containing an sgRNA targeting a specific gene. The sgRNAs first needs to be delivered into a sufficient number of cells via lentivirus or retrovirus transduction. The transduced cells are then grown under specific conditions to select a phenotype of interest. After the selection, the genomic DNA is isolated from the cells, and next-generation sequencing (NGS) performed. The NGS data are then mapped to a pre-complied library comprising the gene-specific gRNAs and the results analyzed by computational tool such as MAGeCK (Model-based Analysis of Genome-wide CRISPR-Cas9 Knockout) (Li et al., 2014). Genes responsible for the observed phenotype are then identified by comparing control and selected gRNAs.
Several studies have now used CRISPR pooled library screening in pancreatic cancer cells (Table 2; Steinhart et al., 2017; Wang et al., 2017; Szlachta et al., 2018; Bakke et al., 2019; Sarr et al., 2019). Bakke et al. (2019) transduced PANC1-cas9 cells with human sgRNA library Brunello and selected the cells with $100 \mathrm{nM}$ gemcitabine for 6 days. PSMA6 was identified as a top hit after comparing NGS results obtained for gemcitabine selected samples and control samples. PSMA6 inhibition was subsequently shown to result in apoptosis and reduced spheroid formation. In Steinhart et al. (2017), a TKO gRNA library was transduced into HPAF-II-Cas9 cells, and cells screened at different time points to identify the set of fitness genes required for proliferation. They used the BAGEL algorithm (Hart and Moffat, 2016) to calculate a log Bayer factor (BF) for each gene. This analysis identified several core components of 
TABLE 2 | Recent researches of CRISPR pooled library screening in pancreatic cancer.

\begin{tabular}{|c|c|c|c|c|c|c|}
\hline Hit genes & Effect & Cell line & Library & Gene/sgRNA number & Selection condition & References \\
\hline PSMA6 & $\begin{array}{l}\text { apoptosis } \uparrow, \\
\text { spheroid } \\
\text { formation } \downarrow\end{array}$ & PANC-1 & $\begin{array}{l}\text { Human sgRNA library Brunello } \\
\text { (addgene No. 73178) }\end{array}$ & $19114 / 76441$ & $\begin{array}{l}100 \text { nM gemcitabine for } \\
6 \text { days }\end{array}$ & Bakke et al., 2019 \\
\hline $\begin{array}{l}\text { Wnt pathway } \\
\text { genes, FZD5 }\end{array}$ & Proliferation $\downarrow$ & $\begin{array}{l}\text { HPAF-II, } \\
\text { AsPC-1, } \\
\text { PaTu8988S }\end{array}$ & $\begin{array}{l}\text { Toronto KnockOut (TKO) CRISPR } \\
\text { Library (addgene } \\
\text { No. 1000000069) }\end{array}$ & $17232 / 91320$ & $\begin{array}{l}\text { different time point (Day 15, } \\
27,31,35)\end{array}$ & Steinhart et al., 2017 \\
\hline \multirow[t]{2}{*}{ CIC, ATXN1L } & $\begin{array}{l}\text { sensitivity to } \\
\text { trametinib } \downarrow\end{array}$ & PATU8902 & $\begin{array}{l}\text { GeCKOv2 library (addgene } \\
\text { No. 1000000049) }\end{array}$ & $19050 / 123411$ & $\begin{array}{l}100 \mathrm{nM} \text { trametinib for } \\
14 \text { days }\end{array}$ & Wang et al., 2017 \\
\hline & & PATU8988T & Avana library Doench et al., 2016 & $18675 / 110257$ & $10 \mathrm{nM}$ trametinib for 14 days & \\
\hline CENPE, RRM1 & $\begin{array}{l}\text { sensitivity to } \\
\text { trametinib } \uparrow\end{array}$ & PDX366 & $\begin{array}{l}\text { Nuclear proteins gRNA sub-pool } \\
\text { library (addgene No. 51047) }\end{array}$ & $7114 / 73151$ & $\begin{array}{l}\text { MEK and CENPE inhibitor } \\
\text { treatment in 6-8-week-old } \\
\text { mail athymic nude mice }\end{array}$ & Szlachta et al., 2018 \\
\hline DCK, DCTPP1 & $\begin{array}{l}\text { sensitivity to } \\
\text { NUC-1031, } \\
\text { gemcitabine } \downarrow\end{array}$ & $\begin{array}{l}\text { Mia PaCa-2, } \\
\text { A2780 }\end{array}$ & $\begin{array}{l}\text { GeCKOv2 library (addgene } \\
\text { No. 1000000049) }\end{array}$ & $19050 / 123411$ & $\begin{array}{l}15 \text { nM gemcitabine or } 65 \mathrm{nM} \\
\text { NUC-1031 for } 14 \text { days or } \\
21 \text { days }\end{array}$ & Sarr et al., 2019 \\
\hline
\end{tabular}

$\uparrow$, up-regulation; and $\downarrow$, down-regulation.

the Wnt pathway, including WLS, CTNNB1, TCF7L2, LRP5, and PORCN, and 3 Frizzled (FZD) receptors and Wnts encoding genes, FZD5, WNT7B, WNT10A, as essential genes for HPAFII cell proliferation. These results were subsequently validated in AsPC-1 and PaTU8988S cell lines, identifying the Wnt pathway as an important mediator of pancreatic cancer cell proliferation. These screen results also revealed that the proliferation and/or survival of RNF43-mutant PDAC cells are selectively dependent on Wnt- $\beta$-catenin signaling. Wang et al. (2017) also performed genome scale CRISPR-Cas9 knockout screens in PATU8902 and PATU8988T cell lines to identify genes capable of promoting proliferation or survival in the context of MAPKi by deletion. They transduced the GeCKOv2 library into PATU8902 cells, and Avana library into PATU8988T cells. PATU8902 cells were treated with MEK1/2 inhibitor trametinib $100 \mathrm{nM}$ and PATU8988T cells were treated with $10 \mathrm{nM}$ trametinib. Both cell lines were treated with trametinib for 14 days. Genomic DNA was then isolated and NGS performed. CIC and ATXN1L were identified by comparative analysis, indicating that loss of both CIC and ATXN1L can reduce sensitivity to trametinib treatment in vivo.

These studies successfully demonstrate that novel gene functions can be identified using CRISPR pooled library screening with specific selection conditions, thereby providing a very powerful methodology for identifying therapeutic vulnerabilities in pancreatic cancer cells and pointing the way toward new treatment strategies. But there are also several factors that can affect the accuracy of the screening results. Based on different size of libraries, different number of cells are required to be transduced for maintaining the coverage of the libraries. Weak selection conditions may provide more comprehensive results, but will also produce false positive which may not be causative in phenotypic changes. Different selection methods may also drive experimental variability. The deep sequencing quality and the selection of data analysis tools will also play very important roles in screening accuracy. Therefore, results still need to be validated using orthogonal methods.

\section{Current Status of CRISPR/Cas9 Gene Therapy for Pancreatic Cancer}

In pancreatic cancer gene therapy, there are several current developments including gene-based tumor cell sensitization to chemotherapy, vaccination, and adoptive immunotherapy (Rouanet et al., 2017). But recently the CRISPR/Cas9 genome editing method has been utilized in several pancreatic cancer studies due to its safety and efficacy. CRISPR/Cas9 gene therapy could be potentially used both ex vivo and in vivo. In ex vivo therapy, cells could be isolated and modified outside of the body, and then transplanted back into the body. In in vivo therapy, genetic materials could be directly injected into the body (Song, 2017). Chiou et al. (2015) built in vivo pancreatic cancer models via retrograde pancreatic ductal injection of either adenoviralCre and lentiviral-Cre vectors. This technique could establish new therapies for pancreatic cancer. Accordingly, CRISPR/Cas9 technology is providing great hope for the treatment of monogenic diseases, degenerative diseases and HIV infection, and several CRISPR/Cas9 clinical trials have been performed, but there is still a long way to go before the CRISPR/Cas9 technique is used to treat pancreatic cancer patients. Several challenges still need to be considered. For example, which crucial gene(s) should be targeted? And the accuracy, efficiency and safety need to be increased to meet the requirement of clinical application.

\section{LIMITATIONS OF THE CRISPR/CAS9 APPLICATION}

CRISPR/Cas9 is widely applied not only in pancreatic cancer research, but also other cancer settings to further our understanding of disease progression, identify mechanisms of drug resistance and to uncover potential therapeutic vulnerabilities. However, there are still some limitations to this technology that need to be considered before CRISPR/Cas9 is used in the clinical practice. Potential off-target effect are a major concern and need to be minimized to ensure safety. Considerable 
effort has been made to reduce off-target effects. Ran et al. (2013) combined the D10A mutant nickase Cas9 (Cas9n) with a pair of offset sgRNAs which are complementary to opposite strands of the target site. This approach improved the specificity of CRISPR/Cas9 editing without sacrificing ontarget cleavage efficiency. Shen et al. (2014) used Cas9(D10A) or Cas9(H840A) to perform AR-A and AR-B gene editing in mouse fibroblast cells. T7EN cleavage assay and TA cloning were performed to detect the modification of the AR gene, and off-target effects were not found. Tsai et al. (2014) demonstrated that dimeric RNA-guided FokI Nucleases (RFNs) could recognize extended sequences and edit endogenous genes with high efficiencies.

\section{CONCLUSION AND OUTLOOK}

Compare to the other gene-editing technologies, CRISPR/Cas9 technology involves lower costs, higher efficiency and simplicity of use. It is widely used to characterize gene function(s) under specific selection conditions and is gaining traction in pancreatic

\section{REFERENCES}

Abdalla, M. Y., Ahmad, I. M., Rachagani, S., Banerjee, K., Thompson, C. M., Maurer, H. C., et al. (2019). Enhancing responsiveness of pancreatic cancer cells to gemcitabine treatment under hypoxia by heme oxygenase-1 inhibition. Transl. Res. 207, 56-69. doi: 10.1016/j.trsl.2018.12.008

Ayars, M., O’Sullivan, E., Macgregor-Das, A., Shindo, K., Kim, H., Borges, M., et al. (2017). IL2RG, identified as overexpressed by RNA-seq profiling of pancreatic intraepithelial neoplasia, mediates pancreatic cancer growth. Oncotarget 8, 83370-83383. doi: 10.18632/oncotarget.19848

Bakke, J., Wright, W. C., Zamora, A. E., Oladimeji, P., Crawford, J. C., Brewer, C. T., et al. (2019). Genome-wide CRISPR screen reveals PSMA6 to be an essential gene in pancreatic cancer cells. BMC Cancer 19:253. doi: 10.1186/s12885-0195455- 1

Barkeer, S., Chugh, S., Karmakar, S., Kaushik, G., Rauth, S., Rachagani, S., et al. (2018). Novel role of O-glycosyltransferases GALNT3 and B3GNT3 in the selfrenewal of pancreatic cancer stem cells. BMC Cancer 18:1157. doi: 10.1186/ s12885-018-5074-2

Barrangou, R., Fremaux, C., Deveau, H., Richards, M., Boyaval, P., Moineau, S., et al. (2007). CRISPR provides acquired resistance against viruses in prokaryotes. Science 315, 1709-1712. doi: 10.1126/science.113 8140

Belvedere, R., Bizzarro, V., Forte, G., Dal Piaz, F., Parente, L., and Petrella, A. (2016). Annexin A1 contributes to pancreatic cancer cell phenotype, behaviour and metastatic potential independently of Formyl Peptide Receptor pathway. Sc.i Rep. 6:29660. doi: 10.1038/srep29660

Chiou, S. H., Winters, I. P., Wang, J., Naranjo, S., Dudgeon, C., Tamburini, F. B., et al. (2015). Pancreatic cancer modeling using retrograde viral vector delivery and in vivo CRISPR/Cas9-mediated somatic genome editing. Genes Dev. 29, 1576-1585. doi: 10.1101/gad.264861.115

Chugh, S., Barkeer, S., Rachagani, S., Nimmakayala, R. K., Perumal, N., Pothuraju, R., et al. (2018). Disruption of C1galt1 gene promotes development and metastasis of pancreatic adenocarcinomas in mice. Gastroenterology 155, 16081624. doi: 10.1053/j.gastro.2018.08.007

Cong, L., Ran, F. A., Cox, D., Lin, S., Barretto, R., Habib, N., et al. (2013). Multiplex genome engineering using CRISPR/Cas systems. Science 339, 819-823. doi: $10.1126 /$ science. 1231143

Doench, J. G., Fusi, N., Sullender, M., Hegde, M., Vaimberg, E. W., Donovan, K. F., et al. (2016). Optimized sgRNA design to maximize activity and minimize offtarget effects of CRISPR-Cas9. Nat. Biotechnol. 34, 184-191. doi: 10.1038/nbt. 3437 cancer research. But there is still a long way before this technology can be used as a selective gene therapy in the clinics. Improving the specificity of cas9 gene editing, reducing off-target effects and increasing the efficiency of cell transduction and cell targeting are all important challenges that need to be overcome. As a maturing gene editing technology, CRISPR/Cas9 represents a "game changer" for scientific research and an exciting targeted therapy for pancreatic cancer.

\section{AUTHOR CONTRIBUTIONS}

HY wrote the manuscript and researched the publication landscape. $\mathrm{PB}$ revised the manuscript. $\mathrm{CP}$ devised the idea.

\section{ACKNOWLEDGMENTS}

The present work was performed in fulfillment of the requirements for obtaining the degree "Dr. Med" of the FAU by HY.

Harada, T., Yamamoto, H., Kishida, S., Kishida, M., Awada, C., Takao, T., et al. (2017). Wnt5b-associated exosomes promote cancer cell migration and proliferation. Cancer Sci. 108, 42-52. doi: 10.1111/cas.13109

Hart, T., and Moffat, J. (2016). BAGEL: a computational framework for identifying essential genes from pooled library screens. BMC Bioinformatics 17:164. doi: 10.1186/s12859-016-1015-8

He, Y., Ye, M., Zhou, L., Shan, Y., Lu, G., Zhou, Y., et al. (2017). High Rab11-FIP4 expression predicts poor prognosis and exhibits tumor promotion in pancreatic cancer. Int. J. Oncol. 50, 396-404. doi: 10.3892/ijo.2016.3828

Hesler, R. A., Huang, J. J., Starr, M. D., Treboschi, V. M., Bernanke, A. G., Nixon, A. B., et al. (2016). TGF-beta-induced stromal CYR61 promotes resistance to gemcitabine in pancreatic ductal adenocarcinoma through downregulation of the nucleoside transporters hENT1 and hCNT3. Carcinogenesis 37, 1041-1051. doi: 10.1093/carcin/bgw093

Hwang, M., Jun, D. W., Kang, E. H., Yoon, K. A., Cheong, H., Kim, Y. H., et al. (2019). EI24, as a component of autophagy. Front. Oncol. 9:652. doi: 10.3389/ fonc. 2019.00652

Ishino, Y., Shinagawa, H., Makino, K., Amemura, M., and Nakata, A. (1987). Nucleotide sequence of the iap gene, responsible for alkaline phosphatase isozyme conversion in Escherichia coli, and identification of the gene product. J. Bacteriol. 169, 5429-5433. doi: 10.1128/jb.169.12.5429-5433.1987

Jinek, M., Chylinski, K., Fonfara, I., Hauer, M., Doudna, J. A., and Charpentier, E. (2012). A programmable dual-RNA-guided DNA endonuclease in adaptive bacterial immunity. Science 337, 816-821. doi: 10.1126/science.1225829

Lal, S., Cheung, E. C., Zarei, M., Preet, R., Chand, S. N., Mambelli-Lisboa, N. C., et al. (2017). CRISPR Knockout of the HuR gene causes a xenograft lethal phenotype. Mol. Cancer Res. 15, 696-707. doi: 10.1158/1541-7786.MCR-160361

Li, M., Hunt, J., Bhattacharyya, A., and Zhao, X. (2019a). One-step generation of seamless luciferase gene knockin using crispr/cas9 genome editing in human pluripotent stem cells. Methods Mol. Biol. 1942, 61-69. doi: 10.1007/978-14939-9080-1_5

Li, M., Xie, H., Liu, Y., Xia, C., Cun, X., Long, Y., et al. (2019b). Knockdown of hypoxia-inducible factor-1 alpha by tumor targeted delivery of CRISPR/Cas9 system suppressed the metastasis of pancreatic cancer. J. Control Release 304, 204-215. doi: 10.1016/j.jconrel.2019.05.019

Li, Z., Shao, C., Liu, X., Lu, X., Jia, X., Zheng, X., et al. (2019c). Oncogenic ERBB2 Aberrations and KRAS mutations cooperate to promote pancreatic ductal adenocarcinoma progression. Carcinogenesis [Epub ahead of print].

Li, W., Xu, H., Xiao, T., Cong, L., Love, M. I., Zhang, F., et al. (2014). MAGeCK enables robust identification of essential genes from genome-scale 
CRISPR/Cas9 knockout screens. Genome Biol. 15:554. doi: 10.1186/s13059014-0554-4

Liu, B., Yang, H., Pilarsky, C., and Weber, G. F. (2018a). The effect of GPRC5a on the proliferation, migration ability, chemotherapy resistance, and phosphorylation of GSK-3beta in pancreatic cancer. Int. J. Mol. Sci. 19:1870. doi: 10.3390/ijms19071870

Liu, B., Yang, H., Taher, L., Denz, A., Grutzmann, R., Pilarsky, C., et al. (2018b). Identification of prognostic biomarkers by combined mRNA and miRNA expression microarray analysis in pancreatic cancer. Transl. Oncol. 11, 700-714. doi: 10.1016/j.tranon.2018.03.003

Liu, X., Huang, Y., Yuan, H., Qi, X., Manjunath, Y., Avella, D., et al. (2019). Disruption of oncogenic liver-intestine cadherin (CDH17) drives apoptotic pancreatic cancer death. Cancer Lett. 454, 204-214. doi: 10.1016/j.canlet.2019. 04.022

Ma, Y., Zhang, L., and Huang, X. (2017). Building cre knockin rat lines using CRISPR/Cas9. Methods Mol. Biol. 1642, 37-52. doi: 10.1007/978-1-49397169-5_3

Mali, P., Yang, L., Esvelt, K. M., Aach, J., Guell, M., DiCarlo, J. E., et al. (2013). RNA-guided human genome engineering via Cas9. Science 339, 823-826. doi: $10.1126 /$ science. 1232033

Muniyan, S., Haridas, D., Chugh, S., Rachagani, S., Lakshmanan, I., Gupta, S., et al. (2016). MUC16 contributes to the metastasis of pancreatic ductal adenocarcinoma through focal adhesion mediated signaling mechanism. Genes Cancer 7, 110-124. doi: 10.18632/genesandcancer.104

Muzumdar, M. D., Chen, P. Y., Dorans, K. J., Chung, K. M., Bhutkar, A., Hong, E., et al. (2017). Survival of pancreatic cancer cells lacking KRAS function. Nat. Commun. 8:1090. doi: 10.1038/s41467-017-00942-5

Osborn, M. J., Belanto, J. J., Tolar, J., and Voytas, D. F. (2016). Gene editing and its application for hematological diseases. Int. J. Hematol. 104, 18-28. doi: 10.1007/s12185-016-2017-z

Pessolano, E., Belvedere, R., Bizzarro, V., Franco, P., Marco, I., Porta, A., et al. (2018). Annexin A1 may induce pancreatic cancer progression as a key player of extracellular vesicles effects as evidenced in the in vitro MIA PaCa-2 model system. Int. J. Mol. Sci. 19, E3878. doi: 10.3390/ijms19123878

Platt, R. J., Chen, S., Zhou, Y., Yim, M. J., Swiech, L., Kempton, H. R., et al. (2014). CRISPR-Cas9 knockin mice for genome editing and cancer modeling. Cell 159, 440-455. doi: 10.1016/j.cell.2014.09.014

Ran, F. A., Hsu, P. D., Lin, C. Y., Gootenberg, J. S., Konermann, S., Trevino, A. E., et al. (2013). Double nicking by RNA-guided CRISPR Cas9 for enhanced genome editing specificity. Cell 154, 1380-1389. doi: 10.1016/j.cell.2013.08.021

Rao, C. V., Janakiram, N. B., Madka, V., Kumar, G., Scott, E. J., Pathuri, G., et al. (2016). Small-molecule inhibition of GCNT3 disrupts mucin biosynthesis and malignant cellular behaviors in pancreatic cancer. Cancer Res. 76, 1965-1974. doi: 10.1158/0008-5472.CAN-15-2820

Rouanet, M., Lebrin, M., Gross, F., Bournet, B., Cordelier, P., and Buscail, L. (2017). Gene therapy for pancreatic cancer: specificity, issues and hopes. Int. J. Mol. Sci. 18, E1231. doi: 10.3390/ijms18061231

Santoro, R., Zanotto, M., Carbone, C., Piro, G., Tortora, G., and Melisi, D. (2018). MEKK3 sustains EMT and stemness in pancreatic cancer by regulating YAP and TAZ transcriptional activity. Anticancer. Res. 38, 1937-1946. doi: 10.21873/ anticanres. 12431

Sarr, A., Bre, J., Um, I. H., Chan, T. H., Mullen, P., Harrison, D. J., et al. (2019). Genome-scale CRISPR/Cas9 screen determines factors modulating sensitivity to ProTide NUC-1031. Sci. Rep. 9:7643. doi: 10.1038/s41598-019-44089-3

Sharma, N. S., Gupta, V. K., Dauer, P., Kesh, K., Hadad, R., Giri, B., et al. (2019). O-GlcNAc modification of Sox2 regulates self-renewal in pancreatic cancer by promoting its stability. Theranostics 9, 3410-3424. doi: 10.7150/thno.32615

Shen, B., Zhang, W., Zhang, J., Zhou, J., Wang, J., Chen, L., et al. (2014). Efficient genome modification by CRISPR-Cas9 nickase with minimal off-target effects. Nat. Methods 11, 399-402. doi: 10.1038/nmeth.2857

Siegel, R. L., Miller, K. D., and Jemal, A. (2019). Cancer statistics, 2019. CA Cancer J. Clin. 69, 7-34. doi: 10.3322/caac. 21551

Song, M. (2017). The CRISPR/Cas9 system: their delivery, in vivo and ex vivo applications and clinical development by startups. Biotechnol. Prog. 33, 10351045. doi: 10.1002/btpr.2484
Steinhart, Z., Pavlovic, Z., Chandrashekhar, M., Hart, T., Wang, X., Zhang, X., et al. (2017). Genome-wide CRISPR screens reveal a Wnt-FZD5 signaling circuit as a druggable vulnerability of RNF43-mutant pancreatic tumors. Nat. Med. 23, 60-68. doi: 10.1038/nm.4219

Stock, K., Borrink, R., Mikesch, J. H., Hansmeier, A., Rehkamper, J., Trautmann, M., et al. (2019). Overexpression and Tyr421-phosphorylation of cortactin is induced by three-dimensional spheroid culturing and contributes to migration and invasion of pancreatic ductal adenocarcinoma (PDAC) cells. Cancer Cell Int. 19:77. doi: 10.1186/s12935-019-0798-x

Szlachta, K., Kuscu, C., Tufan, T., Adair, S. J., Shang, S., Michaels, A. D., et al. (2018). CRISPR knockout screening identifies combinatorial drug targets in pancreatic cancer and models cellular drug response. Nat. Commun. 9:4275. doi: 10.1038/s41467-018-06676-2

Tsai, S. Q., Wyvekens, N., Khayter, C., Foden, J. A., Thapar, V., Reyon, D., et al. (2014). Dimeric CRISPR RNA-guided FokI nucleases for highly specific genome editing. Nat. Biotechnol. 32, 569-576. doi: 10.1038/nbt.2908

Vorvis, C., Hatziapostolou, M., Mahurkar-Joshi, S., Koutsioumpa, M., Williams, J., Donahue, T. R., et al. (2016). Transcriptomic and CRISPR/Cas9 technologies reveal FOXA2 as a tumor suppressor gene in pancreatic cancer. Am. J. Physiol. Gastrointest. Liver Physiol. 310, G1124-G1137. doi: 10.1152/ajpgi.00035.2016

Wang, B., Krall, E. B., Aguirre, A. J., Kim, M., Widlund, H. R., Doshi, M. B., et al. (2017). ATXN1L, CIC, and ETS transcription factors modulate sensitivity to MAPK pathway inhibition. Cell Rep. 18, 1543-1557. doi: 10.1016/j.celrep.2017. 01.031

Wang, S. C., Nassour, I., Xiao, S., Zhang, S., Luo, X., Lee, J., et al. (2018). SWI/SNF component ARID1A restrains pancreatic neoplasia formation. Gut 68, 1259-1270. doi: 10.1136/gutjnl-2017-315490

Watanabe, S., Shimada, S., Akiyama, Y., Ishikawa, Y., Ogura, T., Ogawa, K., et al. (2018). Loss of KDM6A characterizes a poor prognostic subtype of human pancreatic cancer and potentiates HDAC inhibitor lethality. Int. J. Cancer 145, 192-205. doi: 10.1002/ijc.32072

Watanabe, S., Shimada, S., Akiyama, Y., Ishikawa, Y., Ogura, T., Ogawa, K., et al. (2019). Loss of KDM6A characterizes a poor prognostic subtype of human pancreatic cancer and potentiates HDAC inhibitor lethality. Int. J. Cancer 145, 192-205. doi: 10.1002/ijc.32072

Yang, H., Wang, H., Shivalila, C. S., Cheng, A. W., Shi, L., and Jaenisch, R. (2013). One-step generation of mice carrying reporter and conditional alleles by CRISPR/Cas-mediated genome engineering. Cell 154, 1370-1379. doi: 10 . 1016/j.cell.2013.08.022

Yasunaga, K., Ito, T., Miki, M., Ueda, K., Fujiyama, T., Tachibana, Y., et al. (2018). Using CRISPR/Cas9 to Knock out Amylase in Acinar Cells Decreases Pancreatitis-Induced Autophagy. Biomed Res. Int. 2018:8719397. doi: 10.1155/ 2018/8719397

Yu, S., Parameswaran, N., Li, M., Wang, Y., Jackson, M. W., Liu, H., et al. (2017). CRABP-II enhances pancreatic cancer cell migration and invasion by stabilizing interleukin 8 expression. Oncotarget 8, 52432-52444. doi: 10.18632/oncotarget. 14194

Yuza, K., Nakajima, M., Nagahashi, M., Tsuchida, J., Hirose, Y., Miura, K., et al. (2018). Different roles of sphingosine kinase 1 and 2 in pancreatic cancer progression. J. Surg. Res. 232, 186-194. doi: 10.1016/j.jss.2018.06.019

Zhang, D., Li, L., Jiang, H., Knolhoff, B. L., Lockhart, A. C., Wang-Gillam, A., et al. (2017). Constitutive IRAK4 activation underlies poor prognosis and chemoresistance in pancreatic ductal adenocarcinoma. Clin. Cancer Res. 23, 1748-1759. doi: 10.1158/1078-0432.CCR-16-1121

Conflict of Interest: The authors declare that the research was conducted in the absence of any commercial or financial relationships that could be construed as a potential conflict of interest.

Copyright (c) 2019 Yang, Bailey and Pilarsky. This is an open-access article distributed under the terms of the Creative Commons Attribution License (CC BY). The use, distribution or reproduction in other forums is permitted, provided the original author(s) and the copyright owner(s) are credited and that the original publication in this journal is cited, in accordance with accepted academic practice. No use, distribution or reproduction is permitted which does not comply with these terms. 